\title{
Correction
}

\section{Correction: Zhang et al., The RCK2 Domain Uses a Coordination Site Present in Kir Channels to Confer Sodium Sensitivity to Slo2.2 Channels}

In the article "The RCK2 Domain Uses a Coordination Site Present in Kir Channels to Confer Sodium Sensitivity to Slo2.2 Channels" by Zhe Zhang, Avia Rosenhouse-Dantsker, Qiong-Yao Tang, Sergei Noskov, and Diomedes E. Logothetis, which appeared on pages 7554-7562 of the June 2, 2010 issue, the wrong Slack (KCNT1) accession number NP 942057 was cited in the Materials and Methods section. The correct accession number is NM-021853.1 and the Gene ID is 60444. The authors regret any inconvenience caused by this error.

DOI: 10.1523/JNEUROSCI.2166-12.2012 